\title{
The prediction of particle size distribution for the effluent polydisperse particle in staining-dominate deep bed filtration process
}

\author{
DING BinBin ${ }^{1, a}$, LI ChaoLin ${ }^{1, b^{*}}$, Luo HaiJian ${ }^{1, c}$ and Wang YaoWu ${ }^{2, d}$ \\ ${ }^{1}$ Environmental Science and Engineering Research Center, Shenzhen Graduate School, Harbin \\ Institute of Technology, Shenzhen 518055, PR China \\ ${ }^{2}$ Shenzhen Key Laboratory of Urban Planning and Decision Making Simulation, Shenzhen Graduate \\ School, Harbin Institute of Technology, Shenzhen 518055, PR China \\ adingbinbin@hit.edu.cn, ${ }^{b}$ lichaolin@hit.edu.cn, 'luohaijian123@163.com, ${ }^{d}$ wyw68@126.com
}

Keywords: Particle size distribution, Polydisperse, Deep bed filtration.

Abstract. A power law relation between the filtration coefficients of straining and flux through small pores based on percolation theory has been reported. It was a monodisperse particle filtration theory, while the actual colloidal suspension is polydisperse particles. In this study, a formula of particle size distribution for the effluent polydisperse particle in staining-dominate deep bed filtration (DBF) process is proposed based on preliminary results of the monodisperse filtration theory. In order to validate this formula, a numerical staining-dominate DBF model is established to obtain particle size distribution for the effluent polydisperse particle, using the parameters of experimental data set to configure the simulation conditions. The numerical simulation is consistent with the predicted results calculated from the formula. It's indicated that the formula applied for the prediction of particle size distribution for the effluent polydisperse particle in staining-dominate DBF process is relatively accurate and effective.

\section{Introduction}

Colloidal suspension transport in porous media is an essential feature of various natural and industrial processes. These processes include petroleum production ${ }^{[1,2]}$, packed-bed filtration ${ }^{[3,4]}$, microbial transport in porous media ${ }^{[5-7]}$, and formation of riverbeds ${ }^{[8]}$. Simulation of filtration is important for various industrial applications to optimize the operation conditions and minimize costs. Study of colloidal suspension transport in porous media requires a model for describing the porous matrix. Colloidal particles can be removed from porous media through gravity segregation, diffusion, straining, bridging, and electrical forces. The migration and deposition of colloidal particles can be analyzed through several models, such as classic model ${ }^{[9-11]}$ and trajectory analysis model ${ }^{[12-14]}$. Both models exhibit limitations in study of DBF for polydisperse particle.

Percolation ${ }^{[15]}$ is originally derived from filtration, and both processes are similar in many aspects. Therefore, percolation model can be applied to study filtration and obtain accurate results ${ }^{[16-19]}$. Percolation model can also be used to predict the properties of medium ${ }^{[20-22]}$ and is associated with network modeling of transport in porous media. Hao et al. ${ }^{[23]}$ applied percolation theory and random walk in a 2D square network to study staining-dominate DBF; this study proposed two particle capture mechanisms and two power-laws that describe the relationship between the fractions of flow through the small pores and the filtration coefficient. Our previous studies ${ }^{[24-26]}$ investigated the effects of coordination number, capture scheme, and pore size distribution (PSD) on simulation of straining-dominant DBF in 2D networks. The results verify that the two parameters of PSD significantly influence the simulation of power law and the experimental calculation of exponents. The simulated data are consistent with the laboratory test results when proper simulation conditions are used even in 2D networks.

The previous studies are mostly based on monodisperse particle filtration theory, while the actual colloidal suspension is mostly polydisperse particles. Therefore, it is necessary to develop the filtration theory of polydisperse particles. Based on the preliminary results of the monodisperse 
filtration theory, the theoretical formula of effluent particle size distribution for polydisperse particles filtration is deduced, and a set of experimental data is selected to validate the theoretical formula.

\section{Experimental section}

For model validation, a staining-dominate deep bed filtration small scale experimental data sets was selected. It refers to injection of various monodisperse colloidal particle suspensions into porous matrix (laboratory test data of the medium 30/125 ${ }^{[23]}$ ) consisted of packing glass bead with monitored inlet and breakthrough particle concentrations. The colloidal particle size and corresponding $\mathrm{Ce} / \mathrm{C}_{0}$ at the steady-state Ce from the aforementioned laboratory test data of medium 30/125 are shown in Table 1. Assume lognormal distribution for pore sizes, the PSD parameters of porous media based on percolation theory ${ }^{[26]}$, and the results are also listed in Table 1.

Table 1 Particle sizes and the corresponding normalized effluent concentrations and estimated PSD parameters with percolation theory. (from cited reference).

\begin{tabular}{cc}
\hline$r_{s}(\mu \mathrm{m})$ & Medium30/125 \\
\hline 1.57 & 0.9680 \\
2.18 & 0.7990 \\
2.84 & 0.5810 \\
3.17 & 0.1221 \\
4.54 & 0.0003 \\
\hline PSD parameters $\mu$ & 1.799 \\
PSD parameters $\sigma$ & 0.535 \\
\hline
\end{tabular}

\section{Theoretical formula of effluent particle size distribution for polydisperse particles filtration}

In staining-dominate DBF, the injected particles are entrapped in porous media when the particle radius $r_{s}$ is larger than the pore throat radius $r_{p}$. Hence, the first key parameter, namely, fraction of large pores $\left(f_{l}\right)$, can be written in terms of probability density function of pore throat radius $f\left(r_{p}\right)$ as follows:

$$
f_{l}\left(r_{s}\right)=\int_{r_{s}}^{\infty} f\left(r_{p}\right) d r_{p}
$$

The probability of colloidal particle entrapment in pore space relies on pore conductivity. If the flow resistance in a microtubule obeys Poiseuille's law, then the average probability of colloidal particles penetrating the large pores as the probability of conducting particle flow can be calculated as follows:

$$
f_{l}^{*}\left(r_{s}\right)=\frac{\int_{r_{s}}^{\infty} r_{p}^{4} f\left(r_{p}\right) d r_{p}}{\int_{0}^{\infty} r_{p}^{4} f\left(r_{p}\right) d r_{p}}
$$

The $f_{1}^{*}$ at the percolation threshold can be written as

$$
f_{c}^{*}\left(r_{s}\right)=\frac{\int_{r_{s}}^{\infty} r_{p}^{4} f\left(r_{p}\right) d r_{p}}{\int_{0}^{\infty} r_{p}^{4} f\left(r_{p}\right) d r_{p}}
$$

where $f_{c}^{*}$ is the flow-biased percolation threshold, and $r_{s c}$ is the particle radius at the threshold.

The colloidal particles that penetrate the backbones of the infinite cluster can pass through the network media, whereas the particles penetrating the branch connected to the backbone are entrapped at the dangling ends ${ }^{[25]}$ (Fig. 1). Hao Yuan ${ }^{[23]}$ reported a derivation process of formulae for percolation, and this process is explained in Appendix A. Here, we briefly review the derivation process of 
power-law relationship: $\lambda \propto\left(1-f_{l}^{*}\right)^{\beta} f_{l}^{*}>>f_{c}^{*}$. The fraction of flow through the backbones of the infinite clusters may be estimated by using the following equation ${ }^{[27,28]}$ :

$$
B=2 f_{l}^{*}-f_{\text {inf }}^{*}-2 \sqrt{f_{\text {inf }}^{*}\left(f_{l}^{*}-f_{\text {inf }}^{*}\right)}
$$

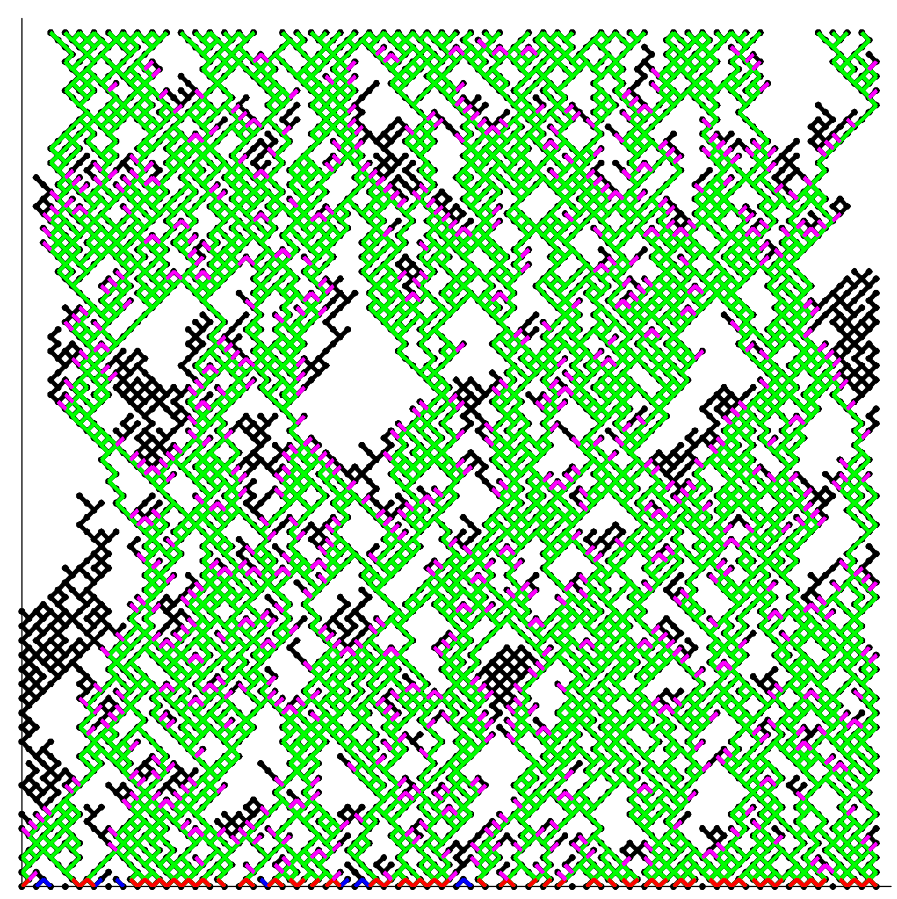

Fig. 1 Cluster structure of the viable pathway, filtration backbone, and branch bonds in the diagonal square lattice (all bonds in the figure are larger than the particle size). Color: green, filtration backbone; purple, branch bonds; black, dangling bonds; red, bonds at the entrance connected to filtration backbone; and blue, bonds at the entrance connected to the dangling bonds).

where $f^{*}$ inf is the fraction of flow through infinite clusters, and B is the fraction of flow through the backbones of the infinite clusters. Far above the percolation threshold, the branch and dangling ends are marginal, and the backbones dominate the main body of the pore network $f^{*}{ }_{\text {inf }} \rightarrow f_{l}^{*}$. The flux through finite clusters and dangling ends can be expressed as:

$$
1-B \approx 1-f_{l}^{*} \quad f_{l}^{*}>>f_{c}^{*}
$$

The distance between two neighboring branches $l_{\mathrm{w}}$ may be assumed to vary by a power law, similar to the power law in Eq. (5):

$$
l_{w} \propto\left(1-f_{l}^{*}\right)^{\gamma} \quad f_{l}^{*}>f_{c}^{*}
$$

where $\gamma$ is the exponent. The filtration coefficient $\lambda$ is defined as the capture probability per unit length of porous medium and can be expressed as:

$$
\lambda \propto \frac{1-B}{l_{w}}=\left(1-f_{l}^{*}\right)^{\beta}
$$

where $\beta$ is power law exponent, which may be determined based on PSD shape, pore structure, and coordination number. Let $\mathrm{K}$ be proportional constant, the $\mathrm{Eq}$ (7) can be rewritten as:

$$
\lambda=K \cdot\left(1-f_{l}^{*}\right)^{\beta} \quad f_{l}^{*}>>f_{c}^{*}
$$

According to the classical theory ${ }^{[29]}$ the filtration coefficient is calculated as $\lambda=-1 / L_{0} \cdot \ln \left(C_{e} / C_{0}\right)$, where $L_{0}$ is the length of column; $\mathrm{C}_{\mathrm{e}}$ and $\mathrm{C}_{0}$ are the lower outlet and influent particle concentration. Combined with Eq (8), the normalized effluent concentrations $\left(\mathrm{C}_{\mathrm{e}} / \mathrm{C}_{0}\right)$ can be derived 


$$
\frac{C_{e}}{C_{0}}=\exp \left[-L_{0} \cdot K \cdot\left(1-f_{l}^{*}\right)^{\beta}\right]
$$

Consider $\mathrm{n}$ challenge tests using monodisperse particles with radii $r_{\mathrm{s} 1}, r_{\mathrm{s} 2} \ldots r_{\mathrm{sn}}$, The inlet and outlet concentrations $\mathrm{C}_{0}\left(r_{\mathrm{si}}\right)$ and $\mathrm{Ce}\left(r_{\mathrm{si}}\right)$ are known from experimental data for each test. Assume lognormal distribution for pore sizes as determined by PSD parameters $\mu$ and $\sigma$. Each experiment data satisfies the following equation:

$$
\frac{C_{e}\left(r_{s i}\right)}{C_{0}\left(r_{s i}\right)}=\exp \left[-L_{0} \cdot K \cdot\left(1-\frac{1+\operatorname{Erf}\left(\frac{\mu+4 \sigma^{2}-\log r_{s i}}{\sqrt{2} \sigma}\right)}{2}\right)^{\beta}\right]
$$

If the particle size distribution of the suspended solids is known, effluent particle size distribution for polydisperse particles filtration can be roughly estimated by combining the monodisperse suspended particles break through curve. The filtration experiment of a polydisperse suspended particles was considered to be the sum of multiple monodisperse experiments. If the particle size distribution function of the influent suspension known as $f\left(r_{s}\right)$, then the particle size distribution formula for polydisperse particles filtration can be expressed as $C_{\mathrm{e}}\left(r_{\mathrm{si}}\right) / C_{0}\left(r_{\mathrm{si}}\right) \times f\left(r_{s i}\right)$, replace with Eq (10), the formula can be rewritten as:

$$
f_{e}\left(r_{s}\right)=f\left(r_{s}\right) \cdot \exp \left[-L_{0} \cdot K \cdot\left(1-\frac{1+\operatorname{Erf}\left(\frac{\mu+4 \sigma^{2}-\log r_{s}}{\sqrt{2} \sigma}\right)}{2}\right)\right]
$$

Where $f\left(r_{s}\right)$ is the particle size distribution function of the known influent suspension, $\beta$ is the theoretical exponent of $\mathrm{Eq}(9)$, the $\mathrm{K}$ is ratio coefficient of $\mathrm{Eq}(9), r_{s}$ is the particle size of any polydisperse suspended particles; $\sigma$ and $\mu$ are pore size distribution parameters of porous media.

\section{Network model setup}

In order to verify the accuracy of this formula, a numerical staining-dominate DBF model is established to obtain particle size distribution for the effluent polydisperse particle, using the parameters of experimental data set (laboratory test data of the medium $30 / 125^{[23]}$ ) to set the simulation conditions. The specific simulation method is similar to that in $\operatorname{Re}^{[23,24]}$., except that the original monodisperse particle size is replaced by the polydisperse particles determined by the particle size distribution function of $f\left(r_{s}\right)$ the influent particle. In this work, we briefly review the procedure of establishing numerical DBF network model.

The trial particles are injected into the network to obtain the effluence probability and the filtration coefficient. The particles that pass through the network are flow biased. The pore clogging and permeability damages of the network are neglected in the simulation and thus the particles walk independently. Particles are injected into the network one by one, and only one particle randomly walks on the lattice each time. Such configurations are used to simulate the particle capture probability of straining mechanism for dilute suspensions. The independent movement of different particles also makes parallel computing possible. Two Xeon E5-2620 processors with $2.0 \mathrm{GHz}$ were applied in parallel computation to perform the simulations. The number of injected particles of the same radius was $10^{5}$ to improve the accuracy of normalized effluent concentrations for filtration simulation.

\section{Results and discussion}

The simulation of staining-dominate DBF model for polydisperse particles was performed in the 
minimum capture scheme by using Square lattice $(100 \times 100)$, and PSD estimated based on percolation theory $(\sigma=1.799$ and $\mu=0.535)$. Assume lognormal distribution for particle size distribution of influent suspension, and the particle size distribution parameters $\mu_{\mathrm{in}}=1.620$ and $\sigma_{\mathrm{in}}=0.53$. Finally, the particles passing through the network media are counted and compared with the predicted results calculated from Eq. (11). As shown in Fig. 2, the results of the numerical simulation (purple curve : effluent polydisperse particle size distribution for simulation) are consistent with the predicted results (black curve: effluent polydisperse particle size distribution for theory) calculated from Eq (11).

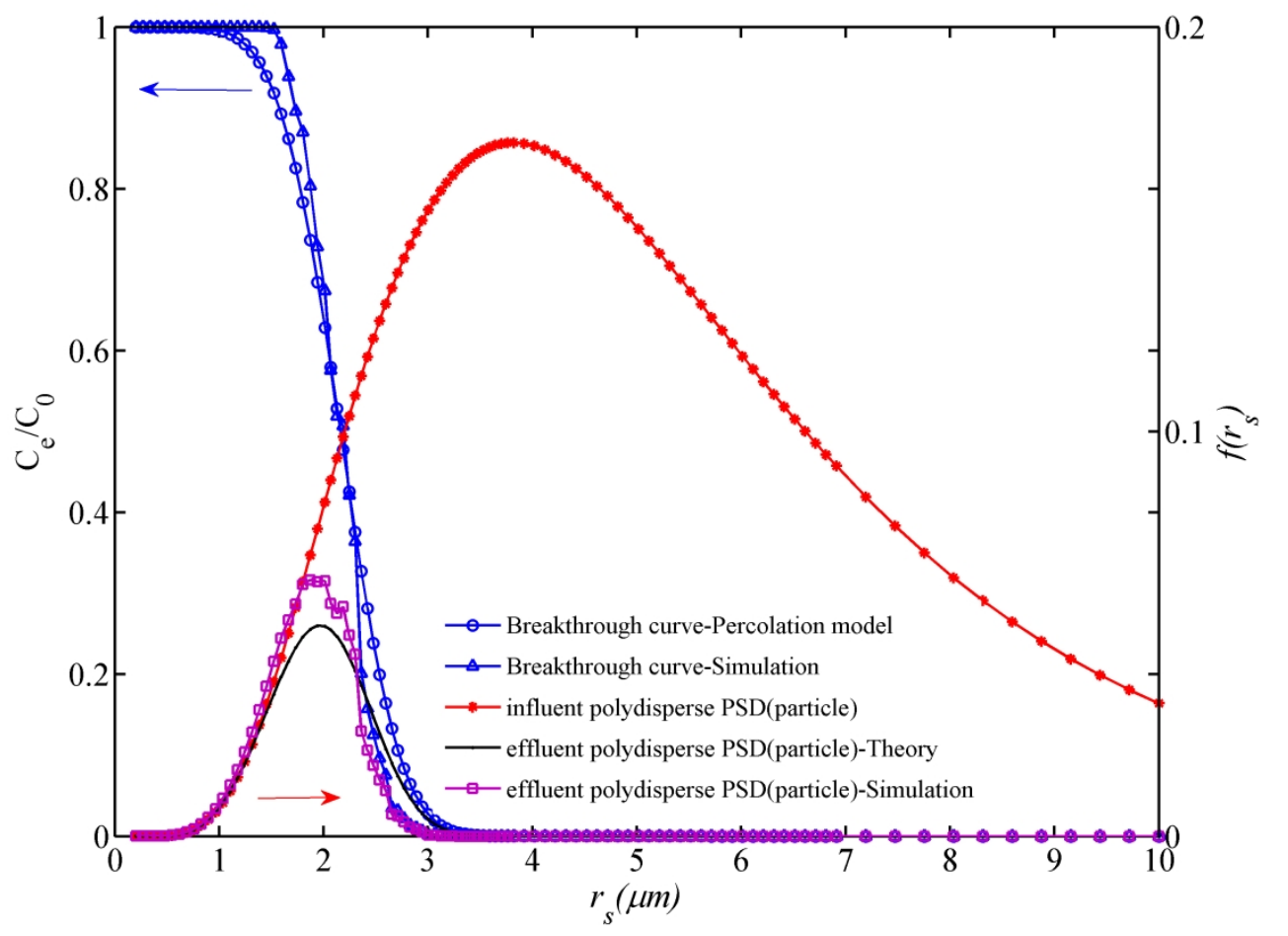

Fig.2 Effluent polydisperse particle size distribution for straining-dominant deep bed filtration estimated by theory calculation and numerical simulation

Since the monodisperse filtration experiments and simulations have neglected the decrease of permeability which caused by pore blockage, thereby the application of this formula also has some limitations: the formula can only be applied to the prediction of the particle size distribution of the effluent of the polydisperse suspended particulates from straining-dominant DBF. If the main mechanism of filtration is not dominated by the straining, the application of the above formula will not yield credible results. Moreover, the calculation and numerical simulation of Eq (11) need to know the PSD of the filter media and particle size distribution of influent particle, otherwise this method cannot be used.

\section{Acknowledgements}

The authors would like to thank Xu Jianhui for valuable discussion and suggestions.

\section{References}

[1] Civan F. Reservoir formation damage : fundamentals, modeling, assessment, and mitigation[J]. Herd Health Environments Research \& Design Journal, 2015,7(2):60-77.

[2] Ding Y. Modeling Formation Damage for Flow Simulations at Reservoir Scale[J]. Spe Journal, 2010,15(3):737-750.

[3] Islam M, Jia X, Fairweather $M$, et al. Prediction of the Permeability and Filtration Performance of Packed Beds[J]. Computer Aided Chemical Engineering, 2011,29(2):1045-1049. 
[4] Chi T, Payatakes A C. Advances in deep bed filtration[J]. Aiche Journal, 1979,25(5):737-759.

[5] Bai H, Cochet N, Drelich A, et al. Comparison of transport between two bacteria in saturated porous media with distinct pore size distribution[J]. Rsc Advances, 2016,6(18):14602-14614.

[6] Tufenkji N. Modeling microbial transport in porous media: Traditional approaches and recent developments[J]. Advances in Water Resources, 2007,30(6-7):1455-1469.

[7] Harvey R W, Kinner N E, Dan M D, et al. Role of physical heterogeneity in the interpretation of small-scale laboratory and field observations of bacteria, microbial-sized microsphere, and bromide transport through aquifer sediments[J]. Water Resources Research, 1993,29(8):2713-2721.

[8] Battin T J, Sengschmitt D. Linking Sediment Biofilms, Hydrodynamics, and River Bed Clogging: Evidence from a Large River[J]. Microbial Ecology, 1999,37(3):185-196.

[9] Ives K J. Deep bed filtration: theory and practice[J]. Filtration and Separation, 1980,17(2):157-166.

[10] Heertjes P M, Lerk C F. The Functioning of Deep bed Filters[J]. Trans. Inst. Chem. Eng, 1967,45:129-145.

[11] Maroudas A, Eisenklam P. Clarification of suspensions: a study of particle deposition in granular media: Part I-Some observations on particle deposition[J]. Chemical Engineering Science, 1965,20(10):867-873.

[12] O'Melia C. Particles, Pretreatment, and Performance in Water Filtration[J]. Journal of Environmental Engineering, 1985,111(6):874-890.

[13] Tien C, Payatakes A C. Advances in deep bed filtration[J]. AIChE Journal, 1979,25(5):737-759.

[14] Yao K, Habibian M T, O'Melia C R. Water and waste water filtration. Concepts and applications[J]. Environmental Science \& Technology, 1971,5(11):1105-1112.

[15] Broadbent S R, Hammersley J M. Percolation processes[M]. 1957.

[16] Bell J D, Deckmyn P, Davies A G. Percolating clusters on Voronoi lattices and the relationship to particle fouling on filters[J]. Zeitschrift für angewandte Mathematik und Mechanik, 1996:599.

[17] Allen H. Relevance of percolation theory to power-law behavior of dynamic processes including transport in disordered media[J]. Complexity, 2009,15(2):13-27.

[18] Golden K M. Percolation Models for Porous Media[M]. Springer New York, 1997.

[19] Ewing R P, Gupta S C. Percolation and permeability in partially structured networks[J]. Water Resources Research, 1993,29(9):3179-3188.

[20] Perrier E M A, Bird N R A, Rieutord T B. Percolation properties of 3-D multiscale pore networks: how connectivity controls soil filtration processes[J]. Biogeosciences Discuss., 2010,7(2):2997-3018.

[21] Berkowitz B, Ewing R P. Percolation theory and network modeling applications in soil physics[J]. Surveys in Geophysics, 1998,19(1):23-72.

[22] Selyakov V I, Kadet V. Percolation Models for Transport in Porous Media: with Applications to Reservoir Engineering[M]. Springer, 1997.

[23] Yuan H, Shapiro A, You Z, et al. Estimating filtration coefficients for straining from percolation and random walk theories[J]. Chemical Engineering Journal, 2012,210(6):63-73.

[24] Ding B, Li C, Zhang M, et al. Effects of pore size distribution and coordination number on the prediction of filtration coefficients for straining from percolation theory[J]. Chemical Engineering Science, 2015,127:40-51.

[25] Ding B, Li C, Dong X. Percolation-based model for straining-dominant deep bed filtration[J]. Separation \& Purification Technology, 2015,147:82-89.

[26] Ding B, Chaolin L. Estimation of pore size distribution of porous media based on percolation theory[J]. CIESC Journal, 2015.

[27] Reyes S, Jensen K F. Estimation of effective transport coefficients in porous solids based on percolation concepts[J]. Chemical Engineering Science, 1985,40(9):1723-1734. 
[28] Larson R G, Davis H T. Conducting backbone in percolating Bethe lattices[J]. Journal of Physics C: Solid State Physics, 1982,15(11):2327.

[29] Santos A, Barros P. Multiple Particle Retention Mechanisms during Filtration in Porous Media[J]. Environmental Science \& Technology, 2010,44(7):7. 